\section{Bridging the species barrier}

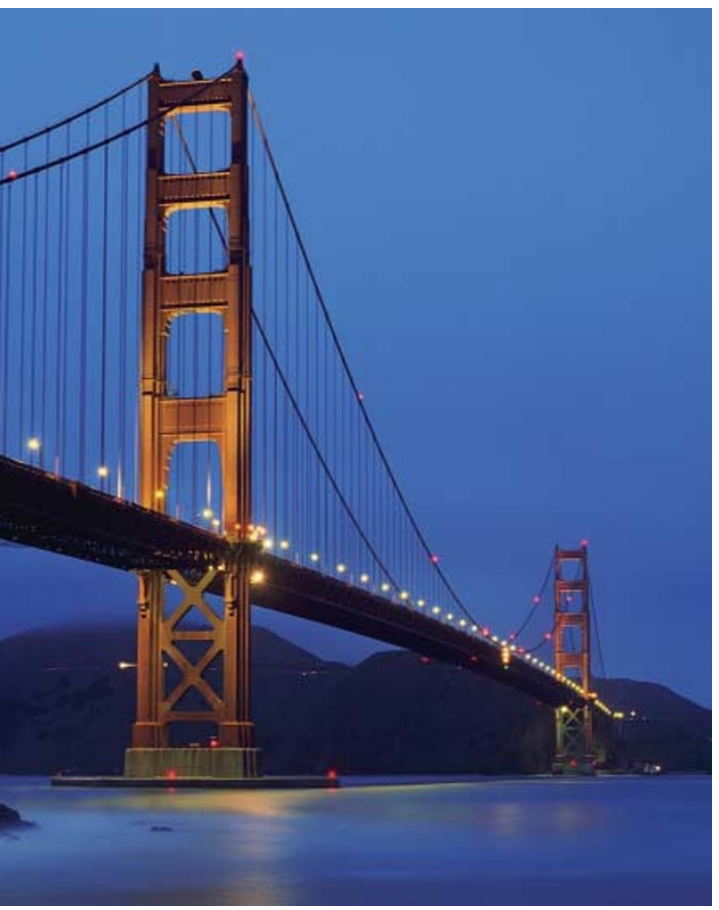

The recent outbreak of avian influenza has led to renewed interest in how avian viruses cross the species barrier and become adapted to humans. But how do avian viruses bind to human receptors, and what made the 1918 influenza virus so infectious? Now, reporting in Science, two groups propose answers to these questions.

The first steps of an influenza infection receptor binding and membrane fusion - are mediated by influenza virus haemagglutinins (HAs), which recognize sialic acids of cellsurface glycoproteins. Sialic acids are present as either $\alpha 2,3$ - or $\alpha 2,6$-linkages - the HAs of avian viruses bind to $\alpha 2,3$-linked sialic acids, whereas those of human viruses bind to $\alpha 2,6$-linked sialic acids. So, HA receptor-binding specificity is important for the virus to infect and spread through the human population.

Reporting in Science, Gamblin et al. and Stevens et al. present X-ray crystal structures of the HA from the 1918 influenza virus (1918HA) - an influenza virus of $\mathrm{H} 1$ subtype that killed more than 20 million people worldwide which reveal several structural surprises. First, the presence of two features that could contribute to the high infectivity of the 1918 virus. The cleavage loop of 1918HA is in a concealed conformation relative to other viral HAs, which could prevent premature cleavage by intracellular proteases, and there are two basic histidine patches that are not observed in other HA structures, which could facilitate membrane fusion in a manner similar to that of vesicular stomatitis virus. Stevens et al. speculate that these could enhance virus infectivity. Second, despite infecting humans, the 1918HA receptor-binding site is characteristic of avian viruses. It is narrower than other human $\mathrm{HAs}$ and, although in $\mathrm{H} 2$ and $\mathrm{H} 3$ virus HAs mutation of Gln226 and Gly228 to Leu and Ser, respectively, have been shown to be associated with changes in binding specificity, the 1918HA has avian residues, similar to other $\mathrm{H} 1$ virus $\mathrm{HAs}$.

So how can this 'avian-type' HA bind to human receptors? Gamblin and co-workers obtained crystal structures of human and avian $\mathrm{H} 1$ and $\mathrm{H} 3 \mathrm{HA}-$ receptor complexes, using $\alpha 2,3$ - and $\alpha 2,6$-linked sialopentasaccharides as avian and human receptor analogues, respectively. Structural analyses identified four residues within the binding site as important for forming interactions with human receptors 190, 193, 222 and 225. The presence of lysine and aspartic acid residues at positions 222 and

\title{
Off the menu
}

The genus Pseudomonas is a physiologically diverse group of bacteria that is present in a wide range of environments, ranging from soil and sewage to plants and animals. A recent report from Environmental Microbiology sheds light on one factor underpinning this ubiquity - resistance to consumption by predatory protozoa.

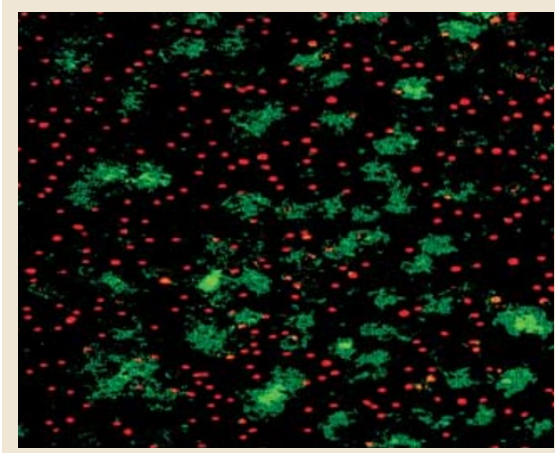

3-day-old biofilm of wild-type $P$. aeruginosa PAO1 (green) growing in the presence of the flagellate $R$. nasuta (red). Image courtesy of Staffan Kjelleberg, University of New South Wales.
The most notorious member of this genus, the opportunistic pathogen Pseudomonas aeruginosa, causes several nosocomial infections. In particular, $P$. aeruginosa, growing as a biofilm, is a major cause of death and morbidity in cystic fibrosis patients. In recent years, it has become well established that biofilm formation is an integral component of the bacterial life cycle, and is a key factor for survival in diverse environments. But do biofilms contribute to the survival of bacteria subject to intense predatory pressure? To investigate this question, Staffan Kjelleberg and colleagues analysed cellular characteristics that are important for $P$. aeruginosa biofilm development for their roles in resisting protozoan feeding.

Using wild-type and mutant strains of the microorganism with altered biofilm development, the authors examined biofilm formation in response to the presence of the common surface-feeding flagellate, Rhynchomonas nasuta. Their initial observations showed that, in the presence of the predator, bacterial microcolonies formed that were resistant to feeding by the flagellate. The authors also tested strains of $P$. aeruginos $a$ that were deficient in quorum sensing, or that lacked type IV pili, and found that these mutant bacteria formed significantly fewer microcolonies compared with the wild-type microorganism. When flagella-deficient $P$. aeruginosa $(\Delta f l i M)$ were exposed to the protozoan, no microcolony formation was observed, indicating a key role for flagella in the development of microcolonies.

Following initial colonization, microcolonies develop into mature biofilms in a differentiation process associated with the production of alginate. When Matz et al. assessed an alginate-overproducing strain of $P$. aeruginosa, the development of larger microcolonies in response to feeding by $R$. nasuta was noted, and the number of protozoa observed was considerably lower than that observed with wild-type bacteria. This finding clearly indicates that alginate production is beneficial for the microorganism as a method of inhibiting predator feeding. The authors were also able to show that mature biofilms formed by wildtype bacteria were acutely toxic to the predator. By contrast, protozoan growth on 
225 , respectively, positions the $\alpha 2,6$-linked sialic acid of a human receptor lower in the HA receptor-binding site then the $\alpha 2,3$-linked sialic acid of an avian receptor. This, in turn, allows Asp190 and Lys222 to form interactions with the receptor, forming a network of interactions between 1918HA residues and human receptors.

These analyses provide important insights into the interactions between HAs and human receptors, and reveal the residues that enable 1918HA to interact with human receptors. All human influenza viruses responsible for major epidemics — including the virus responsible for the 1918 influenza pandemic — are believed to be of avian origin. Although based on a now extinct influenza virus, these analyses could be used to design anti-influenza agents and vaccines to help prevent outbreaks of viral diseases such as avian influenza in humans.

Jane Saunders

\section{(9) References and links}

ORIGINAL RESEARCH PAPERS Gamblin, S. J. et al. The

structure and receptor-binding properties of the 1918 influenza hemagglutinin. Science (5 Feb 2004) doi:10.1126/ science.1093155 | Stevens, J. et al. Structure of the uncleaved human $\mathrm{H} 1$ hemagglutinin from the extinct 1918 influenza virus. Science (5 Feb 2004) doi:10.1126/science.1093373 WEB SITES

Steve Gamblin's laboratory:

http://www.nimr.mrc.ac.uk/protstruct/gamblin/ Ian Wilson's laboratory: http://www.scripps.edu/mb/wilson/

mature biofilms formed by rhlR/lasR mutant bacteria was very efficient. As the RhlR/LasR quorum-sensing systems are known to regulate the expression of several toxins, these findings indicate that quorum-sensingmediated toxin production in mature biofilms is an effective mechanism that allows $P$. aeruginosa to resist protozoan feeding.

In conclusion, the authors have convincingly shown that the formation of microcolonies in the early stages of biofilm development, the production of toxins at later stages, and the regulation of these processes by cell-to-cell communication combine to promote resistance of $P$. aeruginosa to ingestion by protozoan predators. This protection mechanism might also partly explain the widespread presence of this microorganism, and its persistence in both natural and clinical environments.

David O'Connell

(Q) References and links ORIGINAL RESEARCH PAPER Matz, C. et al. Microcolonies, quorum sensing and cytotoxicity determine the survival of Pseudomonas aeruginosa biofilms exposed to protozoan grazing. Environ. Microbiol. 6, 218-226 (2004) FURTHER READING Hall-Stoodley, L., Costerton, J. W. \& Stoodley, P. Bacterial biofilms: from the natural environment to infectious diseases. Nature Rev. Microbiol. 2, 95-108 (2004) WEB SITE

Centre for Marine Biofouling and Bio-Innovation, University of New South Wales: http://www.babs.unsw.edu.au

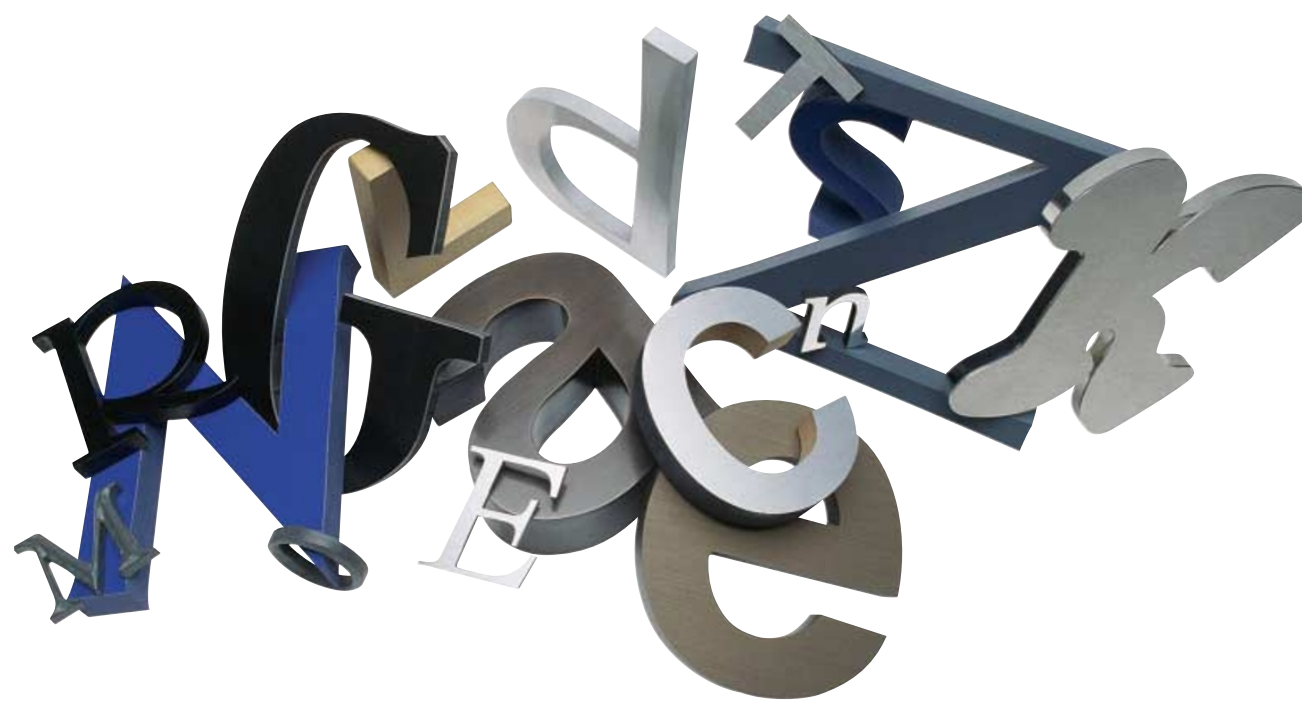

BACTERIAL VIRULENCE

\section{Not quite as easy as $\mathrm{ABC} .$.}

The development of direct genetic analysis techniques for Borrelia burgdorferi is now beginning to give researchers an insight into the specific functions of this spirochete's outer membrane proteins, as three recent publications have shown.

The life cycle of B. burgdorferi, the aetiological agent of Lyme disease in the United States, is divided between mammals and Ixodes scapularis ticks. The ticks ingest spirochetes from an infected mammalian host (commonly the white-footed mouse). B. burgdorferi colonizes the tick mid-gut and remains in a latent state until the tick matures and begins feeding. B. burgdorferi then migrates from the tick midgut to the salivary glands, from where it can be transmitted into a new host.

To survive in such different environments, B. burgdorferi has an extensive repertoire of cell-surface lipoproteins. Until recently, the paucity of direct genetic analysis techniques has hampered the investigation of the precise functions of these proteins. However, three groups have now used direct genetic analysis in B. burgdorferi and have recently reported their results.

Yang et al. targeted the osp $A / B$ gene. Analysis of the infectivity of the resulting deletion mutant in mice and ticks showed that, although OspA/B is not essential for B. burgdorferi to colonize mice, it is essential for colonization of the tick mid-gut.

In a separate study, also a collaboration between Michael Norgard and Erol Fikrig's groups, the gene encoding another outer surface protein, OspC, was disrupted. In ticks, immunoelectron microscopy showed that an OspC deletion mutant was unable to bind to tick salivary glands, supporting earlier thinking that OspC is involved in the migration of B. burgdorferi from the mid-gut to the salivary glands. However, in a third study, this time from Patricia Rosa's group, in which osp $C$ was also disrupted, although OspC was found to be essential for B. burgdorferi to infect mice, the opposite result was obtained for the analysis in ticks, with the OspC deletion mutant able to migrate to the salivary glands.

The different experimental designs could account for these different results. One major difference that is apparent between the two studies is the method used to artificially infect ticks with B. burgdorferi - Pal et al. used direct microinjection into the mid-gut, whereas Grimm et al. infected naive ticks by immersion in exponential-phase cultures of B. burgdorferi. Additionally, Pal et al. deleted the entire $\operatorname{sp} p C$ gene, whereas in the work of Grimm et al., ospC was disrupted rather than deleted. Finally, migration to the salivary glands and transmisson to mice were assessed at different times post-tick infection.

The availability of techniques for the effective genetic manipulation of B. burgdorferi is an exciting development for the field. However, it is clear that, even once these techniques are available, teasing out the roles of specific proteins in the complex life cycle of this spirochete will be far from easy.

Sheilagh Clarkson

\section{(6) References and links}

ORIGINAL RESEARCH PAPERS Yang, X. F. et al. Essential role for OspA/B in the life cycle of the Lyme disease spirochete. J. Exp. Med. 199, 1-9 (2004) | Pal, U. et al. OspC facilitates Borrelia burgdorferi invasion of Ixodes scapularis salivary glands. J. Clin. Invest. 113, 220-230 (2004) | Grimm, D. et al. Outer surface protein $\mathrm{C}$ of the Lyme disease spirochete: a protein induced in ticks for infection of mammals. Proc. Natl Acad. Sci. USA 101, 3142-3147 (2004) 